\title{
Laser-interference Surface Patterning of an AA2024-T3 Alloy: Chemistry and Sub-Surface Microstructure
}

\author{
Donovan Leonard ${ }^{1}$, Harry Meyer, III $^{2}$ and Adrian Sabau ${ }^{2}$ \\ ${ }^{1}$ Oak Ridge National Laboratory, United States, ${ }^{2}$ ORNL, United States
}

Solvent-less surface preparation for joining or coating applications reduces the environmental impact and hazardous waste management of traditional solvent intensive methods. In this study laser interferometry with two beams of a pulsed Nd:YAG laser was used to modify the surface structure and chemistry of an aerospace relevant Al alloy AA2024-T3. Short-pulsed laser surface melting (PLSM) has been used as a surface treatment to enhance corrosion resistance of welded joints of $\mathrm{Al}$ alloys ${ }^{1}$ and produced a more chemically homogenous surface layer, reducing microgalvanic attack from second phases present in the bulk alloy ${ }^{2}$. But, past studies have used beam sizes in the range of 70 to $500 \mathrm{um}$ and have not reported large scale application of the surface structuring and sub-surface microstructural changes.

Utilizing the laser interference induced power profiles, this study explored surface structuring of AA2024-T3 directly from local modulation of laser power intensity (alternating micron regions of high-power and nopower) combined with sample rastering to increase the processed surface area. Surface chemistry of the asreceived, spot and raster laser processed regions was investigated using XPS and correlated with STEM and EDS to monitor the effects of interference angle and raster speed on surface patterning and sub-surface effects. With the laser set-up illustrated in Fig. 1a, 8 shots/spot on the AA2024 surface was compared to the as-received Al surface using both OM and SEM. Figure $1 \mathrm{~b}$ shows a $4 \mathrm{~mm}$ diameter imprint of the laser spot in the 8 shot/spot region. At higher magnification, SEM micrographs showed significant changes in the surface roughness and morphology in the laser processed region when compared to as-received AA2025 sheet. Focused ion beam milling was used to lift a sample from the 8 shot/spot region and the STEM Z-contrast micrograph, shown in Fig. 2, revealed that nanoscale $2^{\text {nd }}$ phase particles contained mostly $\mathrm{Al}, \mathrm{Cu}, \mathrm{Si}, \mathrm{O}$ and Mn but were not present in the first $\sim 1$ um below the processed surface. From quantitative EDS analysis, the elemental composition of this $2^{\text {nd }}$ phase free region suggested nanoscale particles had been dissolved back into the matrix. Figure 2 STEM/EDS of a $2^{\text {nd }}$ phase particle below the laser processed region and one that shows dissolution due to the laser processing again suggest subsurface changes below the AA2024 surface may enhance corrosion resistance..$^{[1][3]}$

\section{References}

${ }^{1}$ P. Ryan, P.B. Prangnell, Grain structure and homogeneity of pulsed laser treated surfaces on Al-aerospace alloys and FSWs, Materials Science and Engineering A 479 (2008) 65-75.

${ }^{2}$ C.P. Chan, T.M. Yue, H.C. Man, The effect of excimer laser surface treatment on the pitting corrosion fatigue behaviour of aluminium alloy 7075, J. Mater. Sci 38 (2003) 2689-2702.

${ }^{3}$ This manuscript has been authored by UT-Battelle, LLC under Contract No. DE-AC05-00OR22725 with the U.S. Department of Energy. The United States Government retains and the publisher, by accepting the article for publication, acknowledges that the United States Government retains a non-exclusive, paid-up, irrevocable, world-wide license to publish or reproduce the published form of this manuscript, or allow others to do so, for United States Government purposes. The Department of Energy will provide public access to these results of federally sponsored research in accordance with the DOE Public Access Plan (http://energy.gov/downloads/doe-public-access-plan). 
(a)
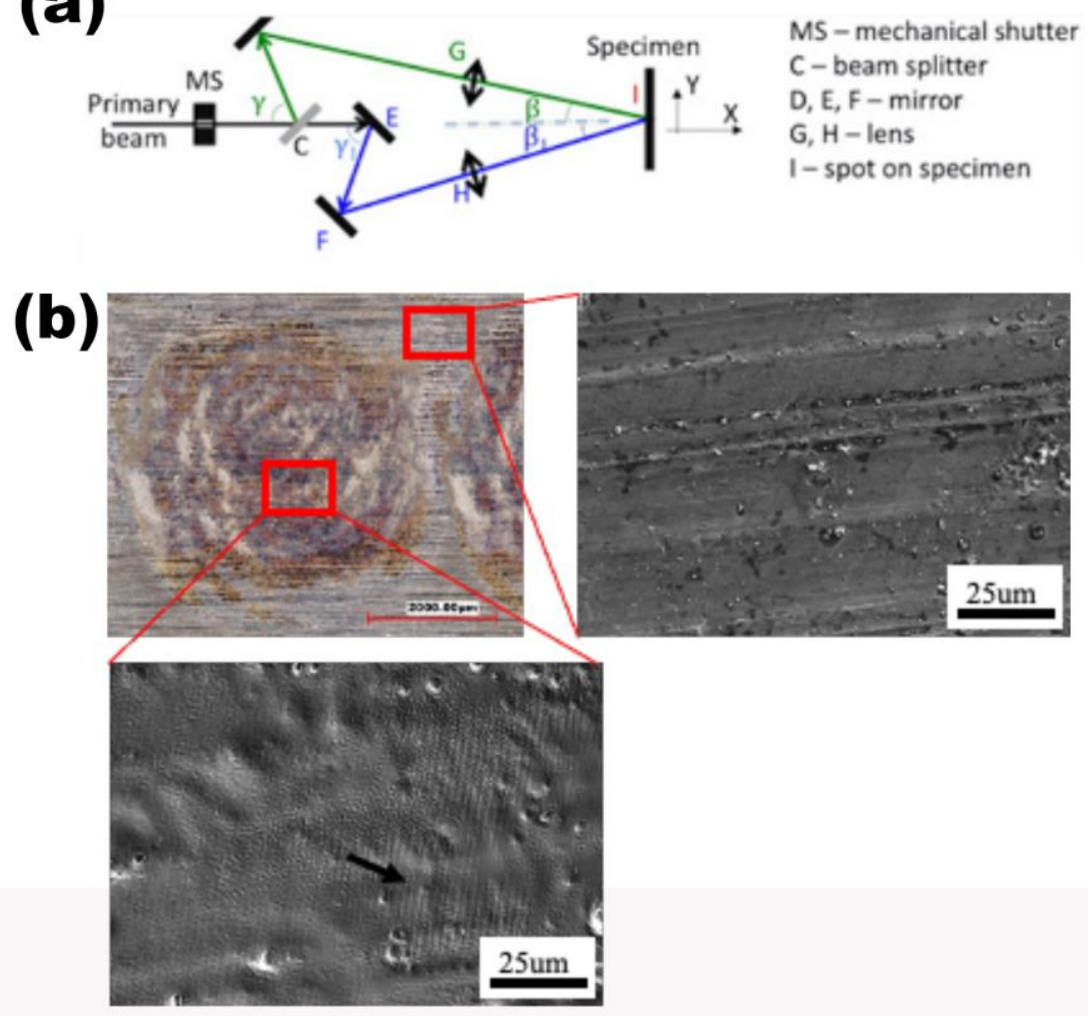

Figure 1. (a) Experimental set up of the laser beam splitting used to modify the AA2024 sample surface. Note the specimen is on a translation stage. (b) (top left) Optical micrograph showing the surface after 8 laser shots. (top right) SEM secondary electron micrograph of as-received surface showing surface asperities produced by rolling. (bottom left) AA2024 surface after 8 shots/spot laser processing, showing surface smoothing and laser interference fringes (arrow).
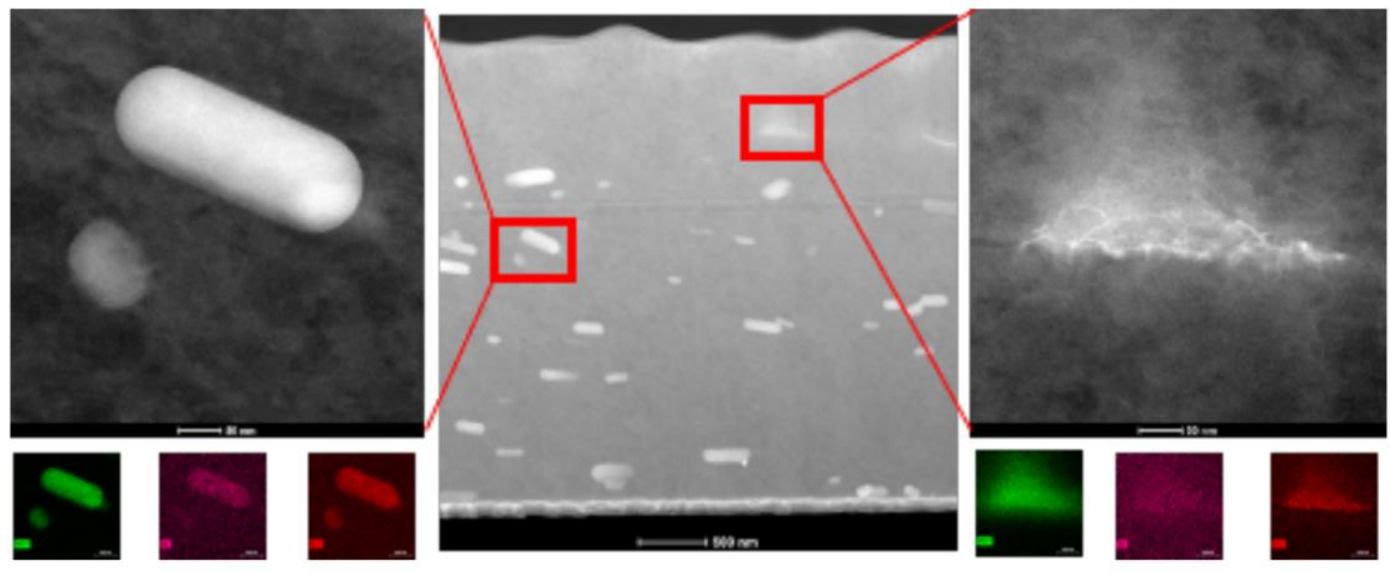

Figure 2. (left) STEM/EDS of 2nd phase nanoparticle not dissolved by laser processing of AA2024 surface. (middle) STEM Z-contrast of AA2024 surface showing dissolution zone 1 um below surface. (right) Dissolving 2nd phase nanoparticle in laser processed region. 